\title{
Dose Proportionality of Treprostinil Sodium Administered by Continuous Subcutaneous and Intravenous Infusion
}

\author{
C. Shane McSwain, MHSc, Ray Benza, MD, Shelley Shapiro, MD, PhD, Nicholas Hill, MD, \\ Robert Schilz, DO, PhD, C. Gregory Elliott, MD, Dianne L. Zwicke, MD, \\ Ronald J. Oudiz, MD, James P. Staszewski, PhD, Carl P. Arneson, MStat, \\ Michael Wade, PhD, David Zaccardelli, PharmD, and Vallerie McLaughlin, MD
}

\begin{abstract}
This study assessed the relationship between dose and plasma concentration following administration of treprostinil sodium infusion therapy in pulmonary arterial hypertension patients. This was a multicenter, open-label, multiple-cohort, steady-state, pharmacokinetic study in subjects with pulmonary arterial hypertension receiving treprostinil by continuous intravenous or subcutaneous infusion at doses between 10 and $125 \mathrm{ng} / \mathrm{kg} / \mathrm{min}$. A blood sample was obtained from each patient at steady state and analyzed via a liquid chromatography/tandem mass spectrometry method. Forty-nine subjects receiving treprostinil were enrolled. Treprostinil doses ranged from 12.1 to $125 \mathrm{ng} /$ $\mathrm{kg} / \mathrm{min}$; treprostinil plasma concentrations ranged from 14.9 to $18248 \mathrm{pg} / \mathrm{mL}$. Linear regression analysis revealed a correlation between treprostinil dose and treprostinil plasma concentration with an $\mathrm{R}^{2}$ value of 0.561. Using a power model to assess dose proportionality, the estimated
\end{abstract}

nonproportionality parameter was 0.641 (95\% confidence interval: 0.083-1.199), reflecting consistency with dose proportionality. Subset linear regression analysis, which excluded 2 subjects with anomalous treprostinil plasma concentrations, increased the $\mathrm{R}^{2}$ value to 0.796. Using a power model to assess dose proportionality of this subset, the estimated nonproportionality parameter was 0.941 (95\% confidence interval: 0.809-1.073). This study supports previous findings of linearity at lower doses up to 15 $\mathrm{ng} / \mathrm{kg} / \mathrm{min}$ and demonstrates linearity at treprostinil doses up to $125 \mathrm{ng} / \mathrm{kg} / \mathrm{min}$.

Keywords: Treprostinil; prostacyclin; pharmacokinetic; pulmonary arterial hypertension (PAH)

Journal of Clinical Pharmacology, 2008;48:19-25 (c) 2008 the American College of Clinical Pharmacology
From the University of Alabama, Birmingham (Dr Benza); West Los Angeles VA Healthcare Center, Los Angeles, California (Dr Shapiro); Tufts-New England Medical Center, Boston, Massachusetts (Dr Hill); University Hospitals of Cleveland, Cleveland, Ohio (Dr Schilz); LDS Hospital and University of Utah, Salt Lake City (Dr Elliott); St. Luke's Medical Center, Milwaukee, Wisconsin (Dr Zwicke); LA Biomedical Research Institute at Harbor-UCLA Medical Center, Torrance, California (Dr Oudiz); United Therapeutics Corporation, Research Triangle Park, North Carolina (Mr McSwain, Dr Staszewski, Mr Arneson, Dr Wade, Dr Zaccardelli); University of Michigan Medical Center, Ann Arbor, Michigan (Dr McLaughlin). Submitted for publication April 2, 2007; revised version accepted September 16, 2007. Address for correspondence: C. Shane McSwain, MHSc, Associate Manager, Clinical Affairs, United Therapeutics Corporation, One Park Drive, 4th Floor, Research Triangle Park, NC 27709; e-mail: csmcswain@unither.com. DOI: $10.1177 / 0091270007309708$
$\mathbf{P}$ rostacyclin, an endogenous substance produced by vascular endothelium, has potent vasodilatory, antiplatelet, and antiproliferative properties. ${ }^{1} \mathrm{~A}$ reduction in prostacyclin urinary metabolites and an increase in thromboxane $A_{2}$ urinary metabolites have been demonstrated in patients with pulmonary arterial hypertension (PAH). ${ }^{2}$ Pulmonary arterial hypertension, defined as an elevation in pulmonary artery pressure and pulmonary vascular resistance in the absence of a secondary cause of pulmonary hypertension, is a severe hemodynamic abnormality that leads to decreased exercise tolerance, dyspnea, and cardiac failure. In patients with PAH, prostacyclin therapy improves pulmonary hemodynamics and functional capacity. ${ }^{3-7}$ 
Treprostinil sodium, a long-acting (terminal half-life of approximately 4.5 hours), ${ }^{8}$ stable, tricyclic benzindene analog of prostacyclin, possesses potent pulmonary and systemic vasodilatory and platelet antiaggregatory actions in vitro and in vivo. ${ }^{9}$ Subcutaneous (SC) treprostinil improved exercise tolerance and indices of hemodynamics, dyspnea, and signs and symptoms of disease in two 12-week clinical trials of 470 subjects with $\mathrm{PAH}^{5}$ and has gained regulatory approval in multiple countries for the treatment of PAH. Recently, treprostinil received regulatory approval as an intravenous (IV) therapy for PAH patients unable to tolerate subcutaneous infusion, based on the bioequivalence of the IV and SC routes of administration. ${ }^{8}$

Treprostinil is delivered by continuous infusion, with doses typically initiated at $1.25 \mathrm{ng} / \mathrm{kg} / \mathrm{min}$ and increased as needed to achieve clinical benefit. ${ }^{9}$ The acute and chronic pharmacokinetics of treprostinil at low doses (1.25-15 ng/kg/min) have been well characterized in studies in normal volunteers ${ }^{8,10,11}$ and in patients with PAH. ${ }^{9}$ These studies demonstrate that the steady-state plasma concentrations of treprostinil range from approximately 30 to $8000 \mathrm{pg} / \mathrm{mL}$ within this dose range and exhibit linear pharmacokinetics (ie, are proportional to dose administered). In a population of patients with inoperable chronic thromboembolic pulmonary hypertension (CTEPH), Skoro-Sajer et $\mathrm{al}^{12}$ observed a proportional relationship between treprostinil dose (range, 11-45 ng/ $\mathrm{kg} / \mathrm{min}$ ) and treprostinil plasma concentration. However, there is little known about treprostinil plasma concentrations at doses above $45 \mathrm{ng} / \mathrm{kg} / \mathrm{min}$, despite the fact that occasional individual patients have been managed effectively on chronic doses greater than $100 \mathrm{ng} / \mathrm{kg} / \mathrm{min}^{13}{ }^{13}$ Therefore, the purpose of this study was to assess whether steady-state treprostinil plasma concentrations remain linearly proportional to doses up to $125 \mathrm{ng} / \mathrm{kg} / \mathrm{min}$.

\section{SUBJECTS AND METHODS}

\section{Study Design}

This was a multicenter, open-label, multiple-cohort, pharmacokinetic study in subjects currently receiving treprostinil by continuous IV or SC infusion for the treatment of PAH. Subjects were receiving treprostinil at a fixed continuous dose between 10 and $125 \mathrm{ng} / \mathrm{kg} / \mathrm{min}$ at least 48 hours after a change in dose. ${ }^{8}$ There were 5 subjects per dose range increment of $10 \mathrm{ng} / \mathrm{kg} / \mathrm{min}$ (ie, 10-19, 20-29, 30-39, 40-49, 50-59,
60-69, 70-79, 80-89, 90-99, and 100-125 ng/kg/min). Following enrollment into the study, 1 steady-state blood sample was obtained from each subject, with the objective of collecting approximately 50 samples collectively. Subjects were monitored for adverse effects related to the pharmacokinetic blood draw prior to being discharged from the study. The study was approved by institutional review boards (IRBs) at each of the 8 participating centers, and written, informed consent was obtained from each subject prior to performing study-related procedures; a list of each IRB is in the appendix.

\section{Pharmacokinetic Parameters and Statistical Analyses}

The primary pharmacokinetic analysis was the correlation between steady-state treprostinil plasma concentrations $\left(\mathrm{C}_{\mathrm{ss}}\right)$ and treprostinil dose. The estimated intersubject variability in steady-state plasma concentrations from previous treprostinil studies (data on file, United Therapeutics Corporation) allowed for adequate precision in the estimated nonproportionality parameter $(\beta)$ from a power model ( $\mathrm{y}=\alpha \mathrm{X}^{\beta}$, where $\mathrm{y}=$ concentration and $\mathrm{X}=$ dose) to detect significant departures from proportionality; under dose proportionality, $\beta=1$. Linear regression analysis was performed on the steady-state treprostinil plasma concentrations versus treprostinil dose. In addition, for descriptive purposes, a simple least squares linear regression model was applied to the steady-state plasma treprostinil concentrations as a function of treprostinil dose.

\section{Subject Population}

Male and female subjects 16 years of age or older, with idiopathic or familial PAH or PAH associated with repaired congenital systemic-to-pulmonary shunts (repaired more than 5 years previously), collagen vascular disease (CVD), or human immunodeficiency virus (HIV), were eligible for the study. Subjects were required to weigh between 45 and $100 \mathrm{~kg}$ and have a body mass index (BMI) between 19 and $29 \mathrm{~kg} / \mathrm{m}^{2}$.

Subjects were excluded if they had a documented history of chronic renal insufficiency (serum creatinine greater than $2.5 \mathrm{mg} / \mathrm{dL}$ or requiring dialysis), hepatic dysfunction (elevation in alanine aminotransferase [ALT] or aspartate aminotransferase [AST] greater than 3 times the upper limit of normal, coagulopathies, abnormal bilirubin, protein and/or platelet counts, as defined by each investigator at each site), or 
anemia (hemoglobin $<10 \mathrm{~g} / \mathrm{dL}$ ) within 3 months of study entry. Because no significant drug interactions have been observed with treprostinil, no concomitant medications were excluded; however, subjects were not eligible for the study if they were receiving an investigational drug, had an investigational device in place, or participated in an investigational drug or device study within 1 month of enrollment.

\section{Treprostinil Sodium Dosing}

Subjects received commercially available continuous SC or IV treprostinil at a dose between 10 and $125 \mathrm{ng} / \mathrm{kg} / \mathrm{min}$. Subcutaneous delivery of treprostinil requires no drug dilution and involves inserting a small bore catheter, typically in the subcutaneous tissue of the anterior abdominal wall, and delivering the medication via a positive-pressure microinfusion pump. For IV administration, treprostinil is diluted in sterile saline and delivered via a permanently placed central venous catheter (eg, Hickman) attached to a portable IV infusion pump.

\section{Blood Sampling}

One 5-mL blood sample was collected from each subject between 1200 and 1700 hours (local time) to minimize the effect of possible diurnal variations in plasma levels during continuous infusion. ${ }^{10}$ The blood samples were collected in tubes containing $\mathrm{K}_{3}$-EDTA as an anticoagulant and immediately placed on ice. Within 1 hour of drawing the blood, the samples were centrifuged at $4^{\circ} \mathrm{C}$ for 10 to $15 \mathrm{~min}$ utes at $3000 \mathrm{~g}$. Plasma separated from the blood was transferred into storage tubes and immediately frozen at $-20^{\circ} \mathrm{C}$ and shipped on dry ice to Prevalere Life Sciences (Whitesboro, New York) for analysis.

\section{Assay Methodology}

A validated liquid chromatography/tandem mass spectrometry (LC/MS/MS) assay was used to determine treprostinil plasma concentrations. The mean percent accuracy values for the quality control samples of the method, run concurrently with the study samples, were $97.3 \%, 99.8 \%$, and $97.8 \%$ of theoretical values with precision (expressed as percent relative standard deviation [RSD]) of $4.83 \%$, $8.56 \%$, and $4.07 \%$ at $30.0,1920$, and $3840 \mathrm{pg} / \mathrm{mL}$, respectively.

Plasma samples were extracted with a mixture of pentane/dichloromethane/isopropanol in a ratio of
45:45:10. The organic phase was separated and evaporated to dryness, and the residual sample was reconstituted in $300 \mu \mathrm{L}$ of 1:1 methanol/water. Samples were analyzed using a reverse-phase C18 analytical column (Thermo Electron Corp, Waltham, Massachusetts; Betasil C18, $5 \mu \mathrm{m}, 2.1 \times 100 \mathrm{~mm}$ ) with a flow rate of $0.4 \mathrm{~mL} / \mathrm{min}$ and an injection volume of $30 \mu \mathrm{L}$. A gradient solvent system was employed with Solvent System A, defined as a 90:10 mixture of water/ammonium acetate $\mathrm{pH} 3.5$ buffer, and Solvent System B, defined as a 90:10 mixture of acetonitrile/ammonium acetate $\mathrm{pH} 3.5$ buffer. A dimethylene homolog of treprostinil (Lot D-101-172-1, purity $99.8 \%$, supplied by UT, Chicago, Illinois) was used as an internal standard. Retention times for treprostinil and the internal standard were approximately 3.2 and 5.0 minutes, respectively.

Detection was done by tandem mass spectrometry (MDS Sciex API 5000), and peak areas were integrated using MDS Sciex Analyst software version 1.4.1. The $\mathrm{m} / \mathrm{z} 389$ to $\mathrm{m} / \mathrm{z} 311$ transition was monitored for the parent compound, and the $\mathrm{m} / \mathrm{z} 417$ to $\mathrm{m} / \mathrm{z} 311$ transition was monitored for the internal standard. Calibration curves were generated from peak area ratios (analyte vs internal standard) using a weighted $(1 / \mathrm{x})$ least squares regression of the ratio compared to the known concentration of the prepared standards. The reliability of the method was determined by duplicate analysis of standards, which were prepared in blank human plasma and extracted using the aforementioned procedure, at 11 concentrations $(10.0,20.0,40.0,80.0,160,320,640,1280$, 2560, 4096, and $5120 \mathrm{pg} / \mathrm{mL}$ ). The lower limit of quantitation (LLOQ) was $10 \mathrm{pg} / \mathrm{mL}$ for a $300-\mu \mathrm{L}$ aliquot of human plasma, and the precision of the method at the LLOQ was $4.35 \%$. Samples with treprostinil concentrations above the limit of quantitation (ALQ, $>5120 \mathrm{pg} / \mathrm{mL}$ ) were diluted 5-fold with human plasma prior to extraction and analysis. Plasma concentrations for these samples were then back-calculated from the standard curve and 5-fold dilution factor. The precision for the dilution integrity quality control samples was $4.84 \%$, and the accuracy was $7.29 \%$.

\section{RESULTS}

Subjects were enrolled between March and August 2006. The demographics of the study subjects are shown in Table I. Forty-three subjects (88\%) were women, and the mean age was 50 (range, 23-80) years old. Eighty-six percent of the subjects were 
Table I Summary of Baseline Demographics and Characteristics

\begin{tabular}{lr}
\hline \hline Parameter & Total (n=49) \\
\hline Age, y & $50.4(23-80)$ \\
Gender & \\
$\quad$ Female & $43(88 \%)$ \\
$\quad$ Male & $6(12 \%)$ \\
Race & \\
$\quad$ Caucasian & $42(86 \%)$ \\
$\quad$ African origin & $5(10 \%)$ \\
Asian & $2(4 \%)$ \\
NYHA class & $7(14 \%)$ \\
I & $25(51 \%)$ \\
II & $16(33 \%)$ \\
III & $1(2 \%)$ \\
IV & $65(46-98)$ \\
Weight, kg & $25(19-40)$ \\
Body mass index, kg/m ${ }^{2}$ & \\
Route of administration & $37(76 \%)$ \\
$\quad$ Subcutaneous & $12(24 \%)$ \\
Intravenous &
\end{tabular}

Data presented as mean (range) or n (\% total). NYHA, New York Heart Association.

Caucasian, 10\% African American, and 4\% Asian. The New York Heart Association (NYHA) functional classification for the study population consisted of 7 (14\%) class I subjects, 25 (51\%) class II, 16 (33\%) class III, and $1(2 \%)$ class IV. The mean weight was $65 \mathrm{~kg}$ (range, 46-98), and the mean BMI was $25 \mathrm{~kg} / \mathrm{m}^{2}$ (range, 19-40). Seventy-six percent of the subjects were receiving treprostinil subcutaneously, whereas $24 \%$ were receiving treprostinil therapy intravenously. Concomitant PAH medication usage for this population consisted of anticoagulants (73\%), diuretics (67\%), phosphodiesterase type 5 inhibitors $(53 \%)$, digoxin $(49 \%)$, endothelin receptor antagonists $(37 \%)$, calcium channel blockers $(24 \%)$, and oxygen ( $8 \%$ ). No adverse events were reported during the study.

Five subjects enrolled in each of the 10 dose range groups (except the 30 - to $39-\mathrm{ng} / \mathrm{kg} / \mathrm{min}$ dosing group, which contained 6 subjects, and the 70- to $79-\mathrm{ng} / \mathrm{kg} /$ min and 100- to $125-\mathrm{ng} / \mathrm{kg} / \mathrm{min}$ dosing groups, which contained 4 subjects each) for a total of 49 subjects. Plasma samples were analyzed and yielded plasma concentrations of treprostinil ranging from 14.9 to $18248 \mathrm{pg} / \mathrm{mL}$. Linear regression analysis produced a fitted line ( $\mathrm{y}=857.4+122.20 \mathrm{x})$ with an $R^{2}$ value of 0.561 (Figure 1). Using the power model to assess dose proportionality, the estimated nonproportionality parameter was 0.641 with a $95 \%$ confidence interval

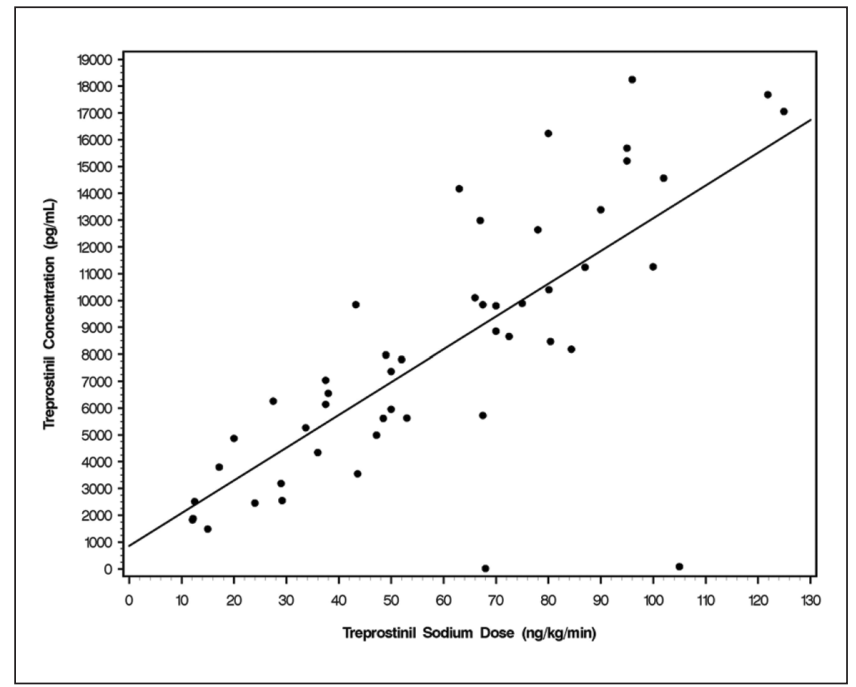

Figure 1. Plot of treprostinil plasma level versus treprostinil sodium dose $(n=49)$.

of 0.083 to 1.199 , reflecting consistency with dose proportionality. This single-sample study in PAH patients exhibited somewhat greater variability in plasma concentration at a given dose level compared to the previous pharmacokinetic (PK) study conducted with normal volunteers in which subjects were given predetermined identical doses of treprostinil at escalating infusion rates. ${ }^{8,10,11}$ However, the results of all these studies are broadly consistent.

The treprostinil plasma levels for 2 subjects (14.9 and $83.8 \mathrm{pg} / \mathrm{mL}$ ) were anomalously low and did not appear consistent with their treprostinil doses, which were 55 and $95 \mathrm{ng} / \mathrm{kg} / \mathrm{min}$, respectively. A spiking experiment, in which known amounts of treprostinil were added to samples of these subjects' plasma, confirmed that there was no substance in these 2 subjects' plasma samples that interfered with the treprostinil assay (data on file, United Therapeutics Corporation). A subset linear regression analysis, which excluded these 2 subjects with aberrant treprostinil plasma concentrations, revealed a fitted line ( $\mathrm{y}=295.3+140.07 \mathrm{x})$ with an $R^{2}$ value of 0.796 (Figure 2). Using the power model to assess dose proportionality of this subset of data, the estimated nonproportionality parameter was 0.941 with a $95 \%$ confidence interval of 0.809 to 1.073 .

\section{DISCUSSION}

Previous studies in healthy volunteers have evaluated the plasma concentrations of treprostinil after dosing with treprostinil sodium and have shown a 


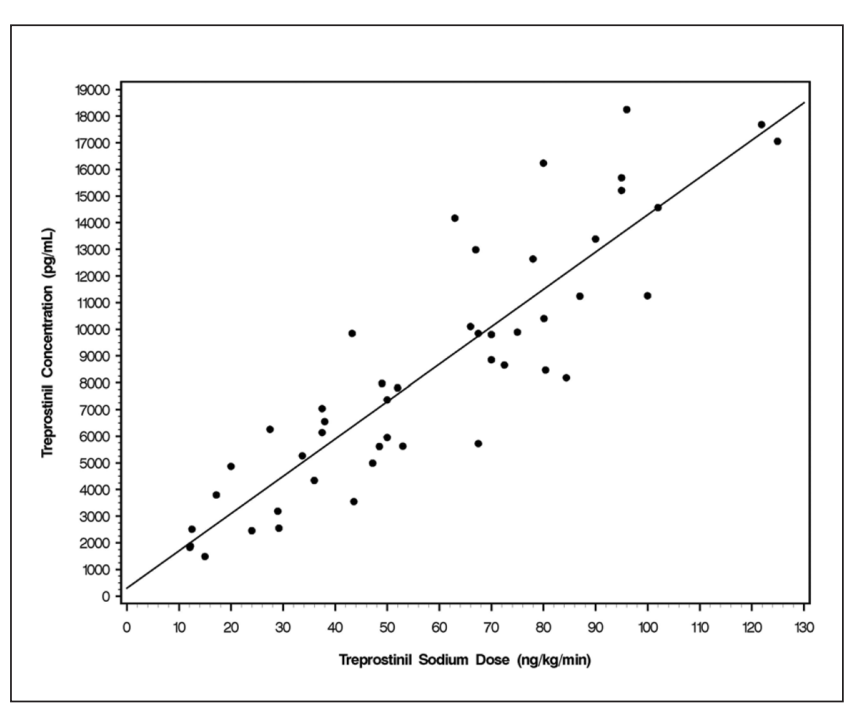

Figure 2. Plot of treprostinil plasma level versus treprostinil sodium dose for subset analysis $(n=47)$.

linear relationship between dose and plasma levels at doses up to $15 \mathrm{ng} / \mathrm{kg} / \mathrm{min} .^{8,10,11}$ In these studies, each incremental dose of $10 \mathrm{ng} / \mathrm{kg} / \mathrm{min}$ produced a corresponding plasma-level increase of approximately $1000 \mathrm{pg} / \mathrm{mL}$. This current study extends these findings and demonstrates a similar dose to plasma-level correlation at therapeutic doses of up to $125 \mathrm{ng} / \mathrm{kg} /$ min. Historically, clinicians have dosed PAH patients with prostacyclins and prostacyclin analogs based on $\mathrm{PAH}$ signs and symptoms while giving consideration to prostacyclin tolerability. Interestingly, SkoroSajer et $\mathrm{al}^{12}$ observed a correlation between treprostinil plasma concentrations and improvements in 6 -minute walk distance $(r=0.497 ; P=.03)$. The additional evidence of proportionality revealed in this study may reduce possible concerns about unpredictable exposure to prostacyclin at higher doses of treprostinil and may also extend the relationship between treprostinil plasma concentration and 6-minute walk distance improvements to higher doses of treprostinil. ${ }^{12}$

For the 2 patients who had anomalously low treprostinil plasma levels, one possibility is that these subjects may produce an uncommonly high level of an active metabolite that is not detected by the assay. Although urinary metabolites of treprostinil have been described, ${ }^{9}$ plasma metabolites have not been characterized, and no treprostinil metabolites have been characterized for pharmacologic activity. It is worth noting that these 2 subjects denied an interruption in treprostinil dosing prior to the blood draws, have responded favorably to treprostinil, and have experienced dose-related symptom changes and treprostinil-associated side effects.

The impact of BMI on treprostinil dosing and the resulting treprostinil plasma levels has not been well characterized in previous studies; however, a recent study in CTEPH patients demonstrated that plasma concentrations of treprostinil are not dependent on SC adipose tissue thickness. ${ }^{12}$ Although this study was not powered for such analyses, in a general linear model in which concentration is predicted simultaneously by dose and BMI, the relationship between dose and concentration was not related to BMI ( $P=.75$ for $\mathrm{n}=49$ [with outliers]; $P=.52$ for $\mathrm{n}=47$ [with outliers excluded]).

Previous research has established that intravenously and subcutaneously administered treprostinil is bioequivalent at steady state. ${ }^{8,10,11}$ The route of administration appears to have no impact on linearity (see Figure 3). A post hoc review of the data, using a general linear model for analysis, revealed that the relationship between dose and concentration was not related to route $(P=.25$ for $\mathrm{n}=49$ [with outliers]; $P=.83$ for $\mathrm{n}=47$ [with outliers excluded]).

It is somewhat problematic to compare this study with previous pharmacokinetic trials of treprostinil. ${ }^{8,10,11}$ By design, this trial consisted of a single blood sample from subjects receiving various treprostinil doses obtained during patient clinic visits, whereas previous pharmacokinetics studies involved samples obtained at prespecified time points from normal healthy volunteers given identical doses and confined to a research clinic during the study period..$^{8,10,11}$ Thus, compared to the earlier trials, the experimental conditions employed in the current study were less controlled, and the subject population was more heterogeneous with regard to a variety of factors, including patient weight and body mass index, diet, concomitant medications, overall health status, and, most important, current administered treprostinil dose. In addition, there were relatively few samples in each dose range cohort in this study. Nonetheless, despite these variables, the data from this study clearly illustrate a linear relationship between treprostinil dose and treprostinil plasma concentrations comparable to that seen in the previous studies and reinforce the current clinical practice of increasing the dose of treprostinil in the presence of progressing PAH symptoms. ${ }^{10}$

\section{CONCLUSION}

This study confirms linearity for treprostinil doses between 10 to $125 \mathrm{ng} / \mathrm{kg} / \mathrm{min}$, and it provides added 


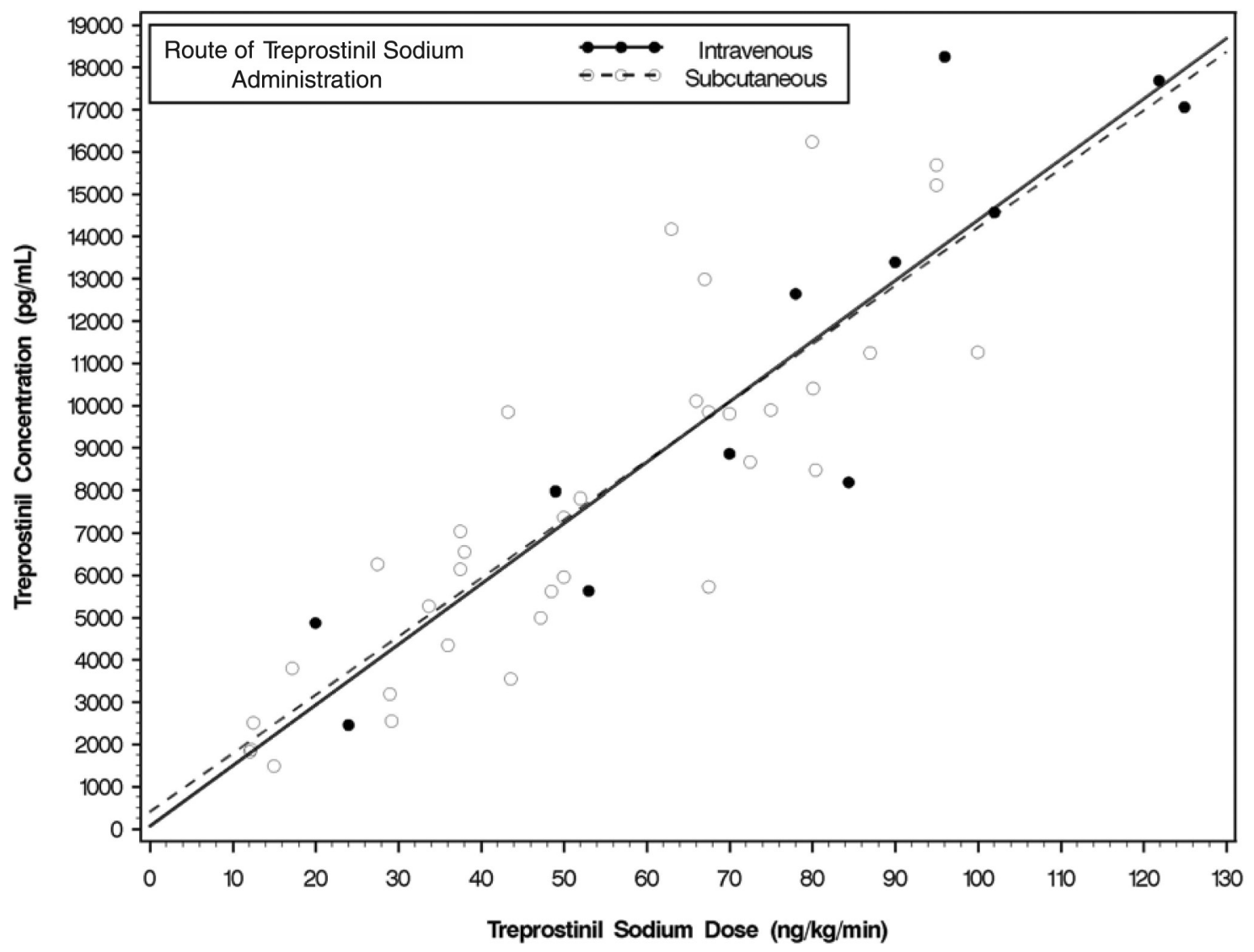

Figure 3. Plot of treprostinil plasma level versus treprostinil sodium dose for subcutaneous and intravenous routes ( $n=47$ ).

assurance that, for the vast majority of patients, an increase in the infusion rate of treprostinil, whether via the SC or IV route, yields a proportional increase in treprostinil plasma concentrations.

The authors gratefully acknowledge the following study coordinators for their efforts on behalf of the study (in alphabetical order): Joy Beckmann, RN (Harbor-UCLA Medical Center, Torrance, California); Raquel Clayton, RN (University of Michigan Medical Center, Ann Arbor, Michigan); Wendy Hill, RN, MS (West Los Angeles VA Healthcare Center, Los Angeles, California); Tamara Monie, RN (Harbor-UCLA Medical Center, Torrance, California); Jessica Robinson, RN (University of Alabama, Birmingham, Alabama); Landra Slaughter, RN (University Hospitals of Cleveland, Cleveland, Ohio); Sandi Stoker, BSN, RN (LDS Hospital and University of Utah, Salt Lake City, Utah); and Karen Visnaw, RN (Tufts-New England Medical Center, Boston, Massachusetts).

Financial disclosure: C. S. McSwain, D. Zaccardelli, J. P. Staszewski, C. P. Arneson, and M. Wade are employed by United Therapeutics. R. Benza and R. Oudiz have received grant support and honoraria from United Therapeutics. S. Shapiro is a consultant for United Therapeutics. N. Hill has received research grants from Actelion, Pfizer, Lilly, United Therapeutics, and Gilead and is a consultant for Pfizer, Actelion, and Gilead. R. Schilz has received grant support, is a consultant, and/or is on the speakers bureau for Merck, Aztra Zeneca, Glaxo-Wellcome, Myogen, Encysive, Actelion, ICOS-Texas Biotechnology, INO Therapeutics, United Therapeutics, and Cotherix. C. G. Elliott is currently or has recently provided consultant services to Actelion, LungRx, Encysive, and United Therapeutics, for which he was paid honoraria. He is a board member for the Pulmonary Hypertension Association, for which he receives reimbursement for his travel expenses. He is a steering committee member for a PAH Registry sponsored by Actelion, for which he received reimbursement for his time as a consultant and travel expenses. His is an employee of Intermountain Healthcare, which currently receives funding from Dr. Elliott's participation as an investigator from Pfizer, Encysive, Actelion, Eli Lilly/ICOS, and United Therapeutics. V. McLaughlin is a consultant for Actelion, Gilead, and Pfizer and has received research grants from Actelion, Pfizer, Encysive, and United Therapeutics. 
APPENDIX

\begin{tabular}{|c|c|c|}
\hline Study Site Name & Study Site Location & Name of Institutional Review Board \\
\hline Harbor-UCLA Medical Center & Torrance, California & John F. Wolf, MD, Human Subjects Committee \\
\hline University of Alabama, Birmingham & Birmingham, Alabama & Western Institutional Review Board \\
\hline LDS Hospital and University of Utah & Salt Lake City, Utah & $\begin{array}{l}\text { Intermountain Health Care Urban Central Region } \\
\text { Institutional Review Board }\end{array}$ \\
\hline University Hospitals of Cleveland & Cleveland, Ohio & $\begin{array}{l}\text { University Hospitals of Cleveland Institutional } \\
\text { Review Board }\end{array}$ \\
\hline Tufts-New England Medical Center & Boston, Massachusetts & $\begin{array}{l}\text { Tufts-New England Medical Center Institutional } \\
\text { Review Board }\end{array}$ \\
\hline West Los Angeles VA Healthcare Center & Los Angeles, California & West Los Angeles VA Institutional Review Board \\
\hline University of Michigan Medical Center & Ann Arbor, Michigan & $\begin{array}{l}\text { University of Michigan Medical School } \\
\text { Institutional Review Board A2 }\end{array}$ \\
\hline St. Luke’s Medical Center & Milwaukee, Wisconsin & Western Institutional Review Board \\
\hline
\end{tabular}

\section{REFERENCES}

1. Vane JR, Botting RM. Pharmacodynamic profile of prostacyclin. Am J Cardiol. 1995;75:3A-10A.

2. Christman BW, McPherson CD, Newman JH, et al. An imbalance between the excretion of thromboxane and prostacyclin metabolites in pulmonary hypertension. $N$ Engl $J$ Med. 1992;327:70-75.

3. Barst RJ, Rubin LJ, Long WA, et al. A comparison of continuous intravenous epoprostenol (prostacyclin) with conventional therapy for primary pulmonary hypertension. The Primary Pulmonary Hypertension Study Group. N Engl J Med. 1996;334:296-302.

4. Badesch DB, Tapson VF, McGoon MD, et al. Continuous intravenous epoprostenol for pulmonary hypertension due to the scleroderma spectrum of disease: a randomized, controlled trial. Ann Intern Med. 2000;132:425-434.

5. Simonneau G, Barst RJ, Galie N, et al. Continuous subcutaneous infusion of treprostinil, a prostacyclin analogue, in patients with pulmonary arterial hypertension: a double-blind, randomized, placebo-controlled trial. Am J Respir Crit Care Med. 2002; 165:800-804.

6. McLaughlin VV, Gaine SP, Barst RJ, et al. Efficacy and safety of treprostinil: an epoprostenol analog for primary pulmonary hypertension. J Cardiovasc Pharmacol. 2003;41:293-299.
7. Tapson VF, Gomberg-Maitland M, McLaughlin VV, et al. Safety and efficacy of IV treprostinil for pulmonary arterial hypertension: a prospective, multicenter, open-label, 12-week trial. Chest. 2006;129:683-688.

8. Laliberte K, Arneson C, Jeffs R, Hunt T, Wade M. Pharmacokinetics and steady-state bioequivalence of treprostinil sodium (Remodulin) administered by the intravenous and subcutaneous route to normal volunteers. J Cardiovasc Pharmacol. 2004;44: 209-214.

9. Remodulin $^{\circledR}$ (treprostinil sodium) [package insert]. Research Triangle Park, NC: United Therapeutics Corporation; 2002.

10. Wade M, Baker FJ, Roscigno R, et al. Pharmacokinetics of treprostinil sodium administered by 28-day chronic continuous subcutaneous infusion. J Clin Pharmacol. 2004;44:503-509.

11. Wade M, Baker FJ, Roscigno R, DellaMaestra W, Hunt TL, Lai AA. Absolute bioavailability and pharmacokinetics of treprostinil sodium administered by acute subcutaneous infusion. J Clin Pharmacol. 2004;44:83-88.

12. Skoro-Sajer N, Bonderman D, Wiesbauer F, et al. Treprostinil for severe inoperable chronic thromboembolic pulmonary hypertension. J Thromb Haemost. 2007;5:483-489.

13. Sitbon O, Manes A, Jais X, et al. Rapid switch from intravenous epoprostenol to intravenous treprostinil in patients with pulmonary arterial hypertension. J Cardiovasc Pharmacol. 2007;49:1-5. 